\title{
Adaptive Pointing - Design and Evaluation of a Precision Enhancing Technique for Absolute Pointing Devices
}

\author{
Werner A. König, Jens Gerken, Stefan Dierdorf, and Harald Reiterer \\ HCI-Group, University of Konstanz \\ Box-D73, Universität Konstanz, 78457 Konstanz, Germany \\ \{koenigw, gerken, dierdorf, reiterer\}@inf.uni-konstanz.de
}

\begin{abstract}
We present Adaptive Pointing, a novel approach to addressing the common problem of accuracy when using absolute pointing devices for distant interaction. First, we discuss extensively some related work concerning the problem-domain of pointing accuracy when using absolute or relative pointing devices. As a result, we introduce a novel classification scheme to more clearly discriminate between different approaches. Second, the Adaptive Pointing technique is presented and described in detail. The intention behind this approach is to improve pointing performance for absolute input devices by implicitly adapting the Control-Display gain to the current user's needs without violating users' mental model of absolute-device operation. Third, we present an experiment comparing Adaptive Pointing with pure absolute pointing using a laser-pointer as an example of an absolute device. The results show that Adaptive Pointing results in a significant improvement compared with absolute pointing in terms of movement time (19\%), error rate (63\%), and user satisfaction.
\end{abstract}

Keywords: Adaptive Pointing, bubble test, pointing precision, hand tremor, control-display gain, distant interaction, laser-pointer.

\section{Introduction}

With the steadily growing diversity of application domains beyond standard desktop usage, absolute pointing devices are becoming more and more favored. Absolute devices use a position-to-position mapping (mouse: velocity-to-velocity) as the transfer function between the input device and the display pointer [1]. As a result the user benefits from a more natural and convenient pointing experience [2] and easier handeye coordination compared with the decoupling of motor and display spaces and the non-linear pointer acceleration when using relative pointing devices. Due to the direct mapping of absolute pointing devices, the user can easily keep track of the cursor, since it is always in line with the user's finger, stylus, laser-pointer or any other absolute device.

Besides home entertainment (e.g. Nintendo Wii), there are various other application domains in, for example, the fields of ubiquitous computing, visual analytics, collaborative environments and interactive exhibitions, where users need the flexibility of absolute pointing devices to interact effectively. Especially in combination with 
large, high-resolution displays, there is a need for input devices that provide more user mobility, allowing the user to work close to the display with detailed information and also to step back and manipulate the contents of the entire display space [3]. This trend is also reflected in research literature, with several authors proposing solutions for absolute input devices such as freehand pointing [3] or laser-pointer interaction $[2,4]$.

However, a common problem shared by all absolute input devices operated from a distance, particularly in combination with high-resolution displays, is the pointing precision. Myers et al. concluded that "interaction techniques using laser-pointers tend to be imprecise, error-prone, and slow" [2]. Vogel et al. reported a similar result for their comparison of absolute, relative and hybrid mapping of hand movements. While the absolute technique was significantly faster than the hybrid and relative ones, the high error rates of the absolute mapping "prevent it from being a practical technique" [3]. Based on previous related work and our experience, we identified two main factors for this serious imprecision of absolute pointing devices used in midair: deviations are caused by natural hand tremor and limited human pointing precision. After discussing these two aspects in detail, we will present the Adaptive Pointing technique, an approach which continuously changes the CD gain of an absolute pointing device to enhance the pointing precision while preserving the behavior of an absolute pointing device.

\subsection{Natural Hand Tremor}

The task of maintaining a part of a limb in a constant position produces involuntary muscular contraction with rhythmical oscillations $(8-40 \mathrm{~Hz})$ referred to as physiological tremor [5]. When using freehand pointing or absolute pointing devices in midair without a stable rest, such natural tremor causes serious noise, which makes accurate pointing and selection more difficult or even impossible as the distance between display and user increases. A variety of approaches exist to reduce noise and so to steady the cursor, such as band-pass filters, dynamic moving windows (Myers et al. [2], Vogel et al. [3]), or using a Kalman filter (e.g. [4]) to smoothen the pointing behavior. While all approaches seem to increase the accuracy they also introduce a noticeable time lag, which reduces the responsiveness of the pointing device. To date, we are not aware of a systematic investigation that compares and ranks these smoothing approaches. All authors report a general improvement, but eliminating noise for pointing movements without introducing a certain amount of delay or reduction of responsiveness seems to be impossible for such reactive methods.iBesides, it is questionable whether even the most perfect jitter compensation would, on its own, provide sufficient pointing accuracy. Thereby another factor has to be considered as well: human pointing precision.

\subsection{Human Pointing Precision}

Absolute pointing devices are characterized by a position-to-position mapping. The user expects that the cursor is in line with the device e.g. laser-pointer. Hence, the pointer motion in display space is proportional to the movement in motor space. When interacting from a greater distance, for example in a presentation situation or 
when using a high-density display, the effective pixel size on the display might fall below human pointing precision. In such a case, even if the tremor compensation worked perfectly, the user would not be able to move discretely one pixel at a time because of limited hand-eye coordination, restricted motor precision, and the necessary but unachievable fine control of the muscle groups involved in the movement (see [6] and [7] for a more detailed discussion). When using a relative input device such as the mouse, the human precision limit can be overcome by lowering the Control-Display gain $\left(\mathrm{CD}\right.$ gain $=$ velocity $_{\text {Pointer }} /$ velocity $\left._{\text {Device }}\right)$ [8]. The CD gain modulates the mapping between the physical input device and the virtual display pointer. With a low-gain transfer function the pointer velocity in display space is several times slower than the actual velocity of the pointing device in motor space. Thus, low CD gain allows for precise targeting even in the case of high-density displays or distant interaction. On the downside, moving long distances is highly inefficient. This speedaccuracy trade-off can be solved by varying the CD gain during interaction. This approach is the basis for several interaction techniques that operate in motor-space and was also the fundamental design principle of our Adaptive Pointing technique.

We will discuss these different techniques according to a classification scheme we have developed. We thus distinguish between target-oriented, manual-switching, and velocity-oriented approaches. Target-oriented techniques basically use a metaphor approach based on magnetism or stickiness by lowering the CD gain when the pointer either enters a target (e.g. [9], [10]) or when it comes close to a target, thus creating a fisheye effect in motor space (e.g. [11], [12]). As a precondition, however, a semantic knowledge of the environment is required, and having to deal with large numbers of targets can be problematic.

The manual-switching approaches rely on the user to manually switch between absolute and relative pointing when appropriate. Forlines et al. [13] rely on this approach with their HybridPointing concept, which provided a two-mode interaction technique with manual switching for pen input on a large, high-resolution display. In this case the switching to relative mode was realized by tapping in a Trailing Widget. Lifting the pen off the display or clicking on the cursor reactivated the absolute mode. They also compared the HybridPointing technique with an exclusively absolute and an exclusively relative pen input. Overall, there was no significant main effect in terms of selection time, but a significant effect on error rate. Hybrid input was worst with $6.8 \%$ versus $4.3 \%$ for the absolute and $3.9 \%$ for the relative mode. The Trailing Widget, which was used for switching the mode, turned out to be "distracting" and sometimes "in the way". Vogel et al. [3] defined different hand postures to explicitly switch between absolute and relative mode in their freehand pointing technique. Hence, the user changes the CD gain manually between a constant value for absolute mode and a conventional acceleration function for relative mode. Vogel et al. compared this two-mode technique named RayToRelative with a solely absolute (RayCasting) and a solely relative mode (Relative). They reported that RayCasting was significantly faster (mean time 2843 ms vs. 3926 ms for Relative and 3744 ms for RayToRelative), particularly so for large targets and when clutching would have been required. However, there was a significantly higher error rate for the absolute RayCasting with a mean error of $22.5 \%$ compared with $3.5 \%$ (Relative) and $5.7 \%$ (RayToRelative). The mean error rate for absolute input even increased to $56 \%$ for the small target $(16 \mathrm{~mm})$ condition. Thus, the combination of absolute and relative mode 
turned out to be a good balance between accuracy and interaction speed. On the downside, the cognitive and physical load of switching explicitly between the two modes remained with the users.

The third group, the velocity-oriented approaches are motivated by the optimizedsubmovement model [14], which states that most aimed movements consist of an initial, large and fast movement towards the target followed by a few slower, corrective movements to compensate for over- or undershooting [15]. The movement velocity in motor space indicates in which phase of the movement the user is and which degree of precision or velocity in display space should be beneficial. This is the basis of all pointer-acceleration techniques already widely in use, for example by default in Mac OS X and Windows XP [16]. In research, different acceleration functions were investigated, for example discrete switches between constant gain levels dependent on the movement velocity, linear acceleration functions, or non-linear mappings. However, the experimental results concerning possible performance improvements in these diverse functions and also in comparison with constant CD gains are inconclusive (see [17] for a detailed discussion). Based on this approach, Frees et al. introduced the PRISM technique which dynamically adjusts the CD gain between the hand and the controlled object in a virtual 3D environment [18]. Evaluation results showed a clear improvement in pointing precision compared to a pure absolute mapping.

The results confirm the impression that combinations of absolute and relative input modes seem to be able to improve pointing precision - but only at a price. The drawback of all these approaches is that an absolute pointing device would no longer maintain the characteristically 1:1 mapping between the device position in motor space and the pointer position in display space. This however would lead to an unnatural and unpredictable behavior. The manual-switching approaches try to resolve this by letting the user choose between absolute and relative mapping while the target-oriented approaches rely on semantic knowledge of the environment, which might not be available. Precise pointing with an absolute input device therefore remains an unsolved problem. In the following section, we discuss our new approach to solving this issue, the Adaptive Pointing technique.

\section{Adaptive Pointing}

We introduce the Adaptive Pointing technique, which can also be classified as a velocity-oriented approach, relying on the optimized-submovement model of Meyer et al. [14] discussed above. However it differs from similar concepts such as PRISM by simulating absolute pointing behavior. The basic idea is to improve pointing performance for absolute input devices by implicitly adapting the CD gain to the current user's needs without violating the users' mental model of absolute-device operation. Users expect a 1:1 mapping between their device movement in motor space and the resulting pointer movement in display space when using an absolute pointing device. Adaptive Pointing appears to provide this pure absolute behavior but imperceptibly lowers the CD gain when higher precision is needed.

While PRISM works very well in the dedicated virtual environment for professional users, it has some obvious drawbacks when applied to a more general setting of (simulating) absolute pointing devices. Since the system explicitly visualizes the 
offset between display space and motor space movement, the device does no longer appear as an absolute pointing device to the user. This also reduces the intuitiveness and ease of use of the device, as the user has to understand at first how this gap between motor space and display space arises and how to deal with it. The absolute pointing behavior is furthermore flawed by the necessary offset reduction. PRISM increases the $\mathrm{CD}$ gain by the amount that is needed so that the offset is nullified within a period of about one second. This, however, should result in a noticeable "jumping" which would lead to an unnatural and unexpected behavior. Furthermore in case of movement direction changes, it might be that the pointer in display space is actually "in front" of the motor space movement. In such a case PRISM lets the users catch up the offset by themselves, which results in a non-movement of the pointer in display space. Again, this behavior results in a reduced ease of use and intuitiveness of the technique when applied to the more generic setting of an absolute pointing device.

Comparing the Adaptive Pointing with the manual-switching approaches, for example [3], [13], the user is not explicitly involved in the gain variation and thus does not need to decide which technique would be most suitable for the next task. Unlike target-oriented approaches such as [11] and [12], Adaptive Pointing does not need any knowledge of the displayed information or active elements. However, it can be easily combined with visual interaction techniques such as expanding targets [19] or Dragand-Pop [20], as well as hand-tremor compensations (e.g. Kalman filter) if further pointing and selection improvement is desired.

\subsection{Adaptive Gain}

The Adaptive Pointing technique dynamically adjusts the CD gain depending on the movement velocity and the current offset between the motor-space position and display-space position. Fig. 1 shows the behavior for the velocity factor. As soon as a predefined minimal velocity threshold is met the CD gain is smoothly decreased. We describe this behavior in the following equations, but only for the horizontal case indicated by the index $x$. Vertical movement is calculated likewise. The first step of the iterative position mapping between motor and display space is the normalization of the velocity, which serves as an indicator of the users' need and as the main controlling factor (Eq. 1). The upper limit is $\mathrm{v}_{\max }$, which marks the threshold from where the CD gain decreases until the lower limit $v_{\min }$ is reached. Velocities below $v_{\min }$ and above $\mathrm{v}_{\max }$ are also limited to a value range of 0 to 1 (Eq. 1). Since we want to ensure an absolute pointing behavior, it is important that the offset between the position in motor space and in display space is considered as well. Eq. 2 describes the offset calculation and the normalization is done likewise to the velocity normalization (Eq. 3). For further calculations we use the larger one of these two factors (Eq. 6). For the special case of a dwelling operation, another factor is added (Eq. 4 \& Eq. 5) to stabilize the cursor even more. Since we want to avoid abrupt switches during the transition from constant gain (absolute mapping) to the varying gain (relative mapping), we use a modulated sine wave as damping function (Eq. 7). When the user decreases speed to aim at a target, the CD gain is smoothly adapted by the modulated sine wave until the minimum gain is reached or the user increases the movement speed again. When the CD gain is lowered, however, the pointer moves more slowly in display 
space than the input device in motor space. This results in an offset between the detected pointing position and the modulated pointer position. In case of either a high velocity or a large offset, the gain calculation reaches values above 1 and up to a predefined maximum.

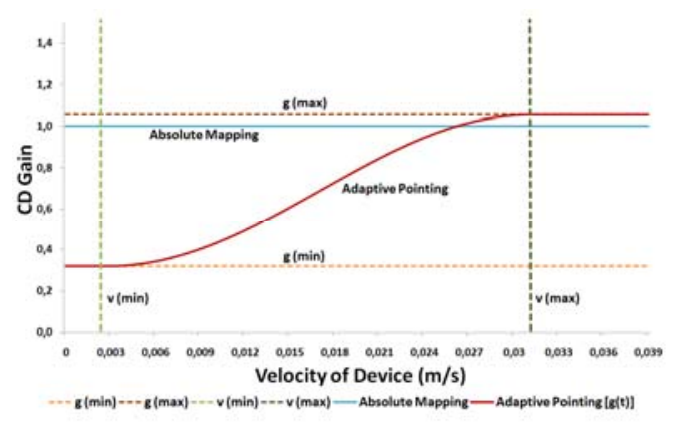

Fig. 1. Smooth transition between relative and absolute CD gain of Adaptive Pointing

In case that the pointer position in display-space lacks behind the position in motor space this results in a smooth catch-up. For the opposite case that the position in display space is "in front" of the position in motor space (e.g. due to a change of direction) we flip the part of the sine wave for which applies CD gain $>1$ at the $\mathrm{CD}$ gain=1.0 axis (Eq. 9). Thereby we reach a gain value slightly below 1 which allows a reverse catch-up of the offset. The new pointer position in display space is then calculated by applying the current $\mathrm{CD}$ gain $\mathrm{g}(\mathrm{t})$ as a factor to the last movement in motor space (Eq. 8) and adding this to the last position $\mathrm{x}_{\text {disp }}(\mathrm{t}-1)$ in display space (Eq. 10).

$$
\begin{aligned}
& \hat{v}_{x}(t)=\left\{\begin{array}{l}
1 \quad \text { if } \quad v_{x}(t)>v_{\max } \\
0 \quad \text { if } v_{x}(t)<v_{\min } \\
\frac{v_{x}(t)-v_{\min }}{v_{\max }-v_{\min }} \text { otherwise }
\end{array}\right. \\
& d_{x}(t)=x_{m o t}(t)-x_{\text {disp }}(t-1) \\
& \hat{d}_{x}(t)=\left\{\begin{array}{l}
1 \quad \text { if }\left|d_{x}(t)\right|>d_{\text {max }} \\
0 \text { if }\left|d_{x}(t)\right|<d_{\text {min }} \\
\frac{\left|d_{x}(t)\right|-d_{\min }}{d_{\max }-d_{\min }} \text { otherwise }
\end{array}\right. \\
& \widehat{w}_{x}(t)=\left\{\begin{array}{l}
1 \quad \text { if } w_{x}(t)>w_{\max } \\
0 \text { if } w_{x}(t)<w_{\min } \\
\frac{w_{x}(t)-w_{\min }}{w_{\max }-w_{\min }} \text { otherwise }
\end{array}\right. \\
& g_{x}(t)=g_{\min }+\frac{1}{2}\left[\sin \left(m_{x}(t) \cdot \pi-\frac{\pi}{2}\right)+1\right]\left(g_{\max }-g_{\min }\right) \\
& \hat{g}_{x}(t)=\left\{\begin{array}{rll}
1-\left(g_{x}(t)-1\right) & \text { if } & g_{x}(t)>1 \text { AND } d_{x}(t)>0 \text { AND } s_{\mathrm{x}}(t)<0 \\
1-\left(g_{x}(t)-1\right) & \text { if } & g_{x}(t)>1 \text { AND } d_{x}(t)<0 \text { AND } s_{x}(t)>0 \\
g_{x}(t) & \text { otherwise }
\end{array}\right. \\
& w_{x}(t)=\frac{1}{\mathrm{n}} \sum_{\mathrm{i}=1}^{\mathrm{n}}\left|x_{m o t}(t)-x_{m o t}(t-i)\right| \\
& \mathrm{m}_{\mathrm{x}}(\mathrm{t})=\widehat{\mathrm{w}}_{\mathrm{x}}(\mathrm{t}) \cdot \max \left(\widehat{\mathrm{v}}_{\mathrm{x}}(\mathrm{t}), \hat{\mathrm{d}}_{\mathrm{x}}(\mathrm{t})\right) \\
& s_{\mathrm{X}}(\mathrm{t})=x_{\text {mot }}(t)-x_{\text {mot }}(t-1) \\
& x_{\text {disp }}(t)=x_{\text {disp }}(t-1)+\hat{g}_{x}(t) \cdot s_{x}(t)
\end{aligned}
$$

This approach allows a smooth and continuous pointer movement that is regulated by parameters for the maximum and minimum values for the $\mathrm{CD}$ gain, the movement velocity, and the offset between display- and motor-space. As pointed out before, this is an important difference to approaches like the PRISM technique, which furthermore does not consider the size of the offset but only the velocity of the movement. We applied the Adaptive Pointing to an infrared laser-pointer interaction system at a 
221 " large high-resolution display (8.9 megapixels Powerwall) to explore the potential as well as the constraints of the novel interaction technique. This is obviously a very demanding setting for absolute pointing techniques, since the user has to point at, select and manipulate very small objects from a distance of several meters (e.g. the Windows start button is only $22 \mathrm{~mm}$ in height on such a display). During iterative testing and configuration we found the following parameters most beneficial for this setting:

$\mathrm{v}_{\min }=0.0028 \mathrm{~m} / \mathrm{s}, \mathrm{v}_{\max }=0.0312^{\mathrm{m}} / \mathrm{s}, \mathrm{d}_{\min }=47 \mathrm{px}, \mathrm{d}_{\max }=232 \mathrm{px}, \mathrm{g}_{\min }=0.032$, and $\mathrm{g}_{\max }$ $=1.055$. Fig. 1 illustrates the resulting $\mathrm{CD}$ gain with respect to the velocity of the input device in motor space for the parameter set used.

\section{Evaluation}

To evaluate the Adaptive Pointing technique we conducted a controlled experiment with 24 participants. As a popular representative of an absolute pointing device we used an infrared laser-pointer interaction technology that is described in more detail in [21]. We compared the Adaptive Pointing technique with a Kalman filter enhanced absolute pointing in terms of efficiency, effectiveness and user satisfaction. In addition, we wanted to compare novices with experienced users of the laser-pointer interaction technology to assess whether the usefulness of the Adaptive Pointing technique diminishes with increasing familiarity with the device.

\subsection{Materials and Participants}

The experiment was conducted in front of the previously mentioned Powerwall (see Fig. 2), a wall-sized display with a resolution of $4640 \times 1920$ pixels and physical dimensions of $5.20 \times 2.15$ meters. The infrared (and thereby invisible) laser-pointer interaction technology is used to interact freely with the display. We applied a combination of static and dynamic Kalman filters for the absolute pointing condition; while for the Adaptive Pointing technique we relied solely on a static Kalman filter since the Adaptive Pointing technique replaces the dynamic component. In both cases we optimized the performance as well as the 'feeling' of the laser-pointer by iterative testing and configuration. The laser-pointer was equipped with a button, which was

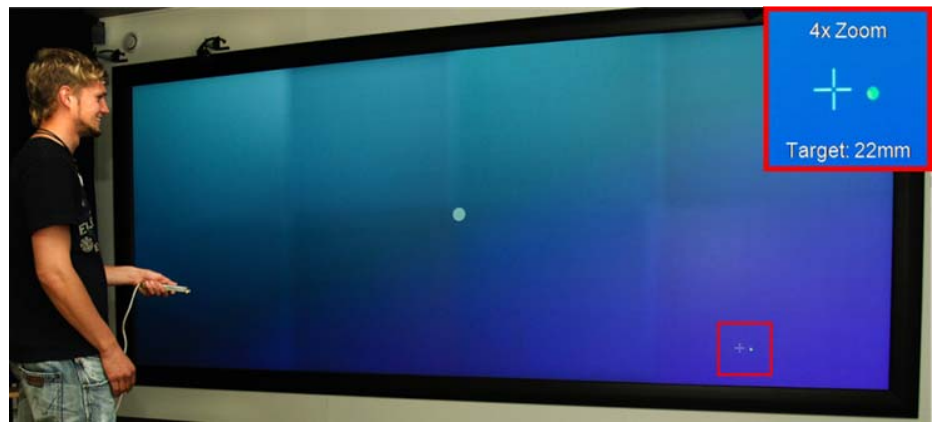

Fig. 2. Comparing absolute input and Adaptive Pointing at a large, high-resolution display. Device: infrared laser-pointer, distance: 3 meters. 
used to click on a target. Demographic data was collected via a pre-test questionnaire. A questionnaire/interview combination was used to assess users' subjective opinions about the Adaptive Pointing technique.

For the study we selected 24 subjects; 16 female and 8 male. Their ages ranged from 16 to 53 years (mean 26.75, st.dev. 8.81 years). Their fields of occupation varied greatly, embracing school pupils, university students and employees. Twelve participants formed the experienced group. They qualified for this group by having already used the laser-pointer with absolute pointing extensively in an earlier study (it took place on average 52.17 days earlier, 3.01 days st. dev.). None of the other twelve subjects (novice group) had ever used an interactive laser-pointer before.

\subsection{Tasks}

We used a 'bubble' task that essentially implements a discrete, multidirectional tapping paradigm to assess the pointing performance of the two different techniques (see Fig. 2). In such a task, users had to move the cursor (in the form of a cross-hair) onto a randomly appearing bubble target and click the button while over it. Between each trial users had to dwell on a homing position located in the center of the screen until the next target appeared. The task is largely along the lines of Fitts' Law experiments, as recommended by ISO-9241-9 with the differences being the use of a discrete tapping paradigm and the use of colors and sounds for motivational reasons (see [22] for a discussion). We used target widths (W) of 20, 40 and 80 pixels (22.4, 44.8 and 89.6 $\mathrm{mm}$ respectively). These appeared in home-to-target amplitudes (A) of 400, 1000, and 1800 pixels. An initial task fulfilled the dual roles of retention task for the experienced group and training task for the novice group. In this case we used target sizes of 40, 80 and 160 pixels and a different color setting to distinguish this training task from the experimental task. Participant used only the absolute pointing technique in this phase. Similar to [2] we used an additional dwelling task in order to assess the steadiness of the Adaptive Pointing technique, i.e. the stability with which one can hold a certain position. Users had to point at a 20-pixel target located in the center of the screen for five seconds, while measuring started one second after first crossing the target border. Each second was indicated with a short 'beep' sound.

\subsection{Experimental Design}

We used a $2 \times 2 \times 3$ split-plot design, the first being a between-subjects factor (experience) and the latter two within-subjects factors (pointing technique, type of task). We fully counter-balanced the pointing-technique factor across the two experimental tasks (bubble + dwelling), leaving the training/retention task at the beginning unaffected, of course. This resulted in four different experimental groups to which we randomly assigned six participants each. The dependent variables were error rate (hit or miss), movement time (time between leaving the homing position and clicking on a target), and subjective rating of the technique (on a 6-point scale in terms of improvement or worsening, depending on the sequence of presenting the pointing techniques). We used of $3 \mathrm{~W}$ (width) $\times 3 \mathrm{~A}$ (amplitude) $\times 16$ trials $\times 2$ blocks and an additional short familiarization phase of $3 \mathrm{~W} \times 3 \mathrm{~A} \times 5$ trials for each of the two pointing technique conditions. Together with 216 training/retention trials this sums up to 882 trials per participant and 21,168 trials in total. The dwelling task was repeated five times by each user for each pointing technique. Fig. 3 illustrates the exact procedure. 


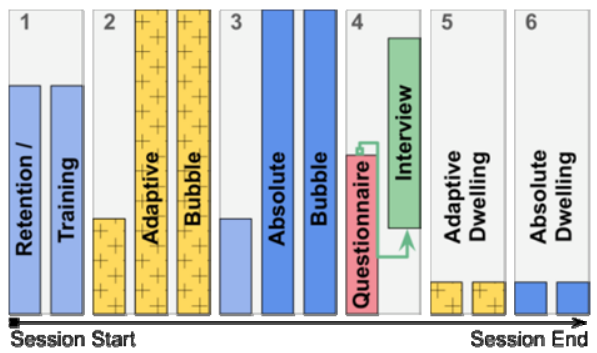

Fig. 3. Illustrating the (counter-balanced) experimental procedure - each pointing technique consisted of one familiarization block and two experimental blocks

It is important to note that participants were not informed of the condition change between absolute and adaptive pointing. Between each block, participants were able to relax for about one minute. After completing all tasks the participants were then compensated for their efforts with a payment of 8 Euros. Each session lasted about 70-90 min.

\subsection{Hypotheses}

Based on our goals and design principles for the Adaptive Pointing technique we formulated the following hypotheses for our experiment. We hereby focused on the general measures of movement time and error rate to be able to distinguish between accuracy and efficiency.

H1: Accuracy - aiming and hitting. Using the Adaptive Pointing technique will enable better aiming at and hitting of targets compared with using absolute pointing. This will become evident in a lower error rate during the bubble task and in lower deviations from the target during the dwelling task. When aiming at a target, users will slow down their movement and thereby enabling the Adaptive Pointing technique. When using absolute pointing, earlier studies suggest error rates of about $15 \%$ [21] and dwelling deviations between 7.3 and $8.9 \mathrm{~mm}$ [2].

H2: Moving. Regarding the movement time we expected the Adaptive Pointing technique to perform on a level comparable with absolute pointing. Since moving long distances is normally done at a higher speed [14], the CD gain should remain comparable with pure absolute pointing and therefore not affect the movement. However, since the measure 'movement time' includes the time for actually aiming and clicking, we expected the movement time for small targets to be lower compared with absolute pointing. The latter should need more time in the aiming phase in order to achieve a hit, especially when the targets are only 20 pixels in width.

H3: Imperceptibility. Since participants were not instructed of the change in conditions between absolute and adaptive pointing, we assumed that participants would either not recognize a change in the behavior of the laser-pointer or not ascribe it to the laser-pointer itself. The post-test questionnaire explicitly asked about any kinds of change noticed during the experiment in terms of accuracy, ease of use, and performance as well as the reasons that people claimed to be responsible for these changes. 
One design rationale behind the Adaptive Pointing was to integrate an imperceptible change in $\mathrm{CD}$ gain, preserving the feeling of a pure absolute pointing device. According to a study by Sutter et al. [23], people tend to judge their hand movement mainly on the basis of the on-screen movement of the cursor and adapt their hand movement accordingly. This means that, as long as the discrepancy between cursor position and hand position is quite small, people will not recognize any discrepancy at all and therefore will not ascribe the different accuracies of per-se absolute pointing devices to the devices themselves.

H4: Experience. This last hypothesis assumed that people with more experience would 1) perform better than the novice group and 2) benefit less from the Adaptive Pointing in terms of the first two hypotheses. While the first point should be due to the larger amount of training producing positive results, as has been discovered before (see [22]), the second point reflects the thinking that a higher level of performance (due to training) naturally leads to less room for improvement.

\section{Results}

For further analysis and testing of our hypotheses we considered the 13,824 trials during the bubble task. In a first step, we removed $1.7 \%$ of these trials after identifying them as either accidental clicks or extreme outliers resulting in 13,578 trials used for analysis. Furthermore, one participant (in the experienced group) was completely excluded because of error rates higher than $25 \%$ regardless of the pointing technique. During the interview he stated that he didn't really try to hit the targets. Homogeneity of variances was met in all cases when contrasts or pair-wise comparisons were performed.

H4: experience: We begin the presentation of the results with our last hypothesis, which stated that experienced users would perform better, and benefit less from the Adaptive Pointing technique, when compared with the novice group. Results, however, show that both groups performed fairly equally. Table 1 shows that the small differences are non-significant.

Table 1. Comparing experience levels

\begin{tabular}{l|l|l} 
& Error rate/std.err. (in \%) & Movement time / std. err. (in s) \\
\hline Novice & $11.26 / 1.49$ & $1.67 / 0.74$ \\
\hline Expert & $8.8 / 1.45$ & $1.72 / 0.87$ \\
\hline F-statistic & $\mathrm{F}(1,21)=1.365, \mathrm{p}=0.256$ & $\mathrm{~F}(1,21)=0.430, \mathrm{p}=0.519$
\end{tabular}

We therefore have to withdraw the hypothesis in favor of the null-hypothesis. This result is somewhat surprising. An analysis of the retention task in comparison to the earlier study reveals that although the performance of the experienced participants decreased about 5\%, they were still superior to the novice group (about 16\%), although the difference is not significant $(\mathrm{p}=0.069)$. Nevertheless, this might indicate that the increased difficulty of the bubble task made the training obsolete. In future 
studies we will investigate the influence of task difficulty on learning more in detail. Based on these findings we do not report the following findings with respect to different experience levels.

H1: accuracy (aiming \& hitting): We first analyzed the error rate during the bubble task. A 2 (exp.) $\times 2$ (pointing technique) Repeated-Measures(RM)-ANOVA (measure: error rate) shows a significant main effect for pointing technique $(\mathrm{F}(2,20)=42.836$, $\mathrm{p}=0.000$ ). Post-hoc pair-wise comparisons (with Bonferroni adjustments) reveal that the error rates differ significantly in favor of the Adaptive Pointing technique $(5.4 \%$ compared with $14.77 \%$, std. err.: $0.7 \%$ for adaptive and $1.79 \%$ for absolute, $\mathrm{p}=0.000$; confidence intervals (95\%) for error rate: 3.93\%-6.82\% (adaptive) vs. $12.24 \%$ $17.13 \%$ (absolute)). Given the confidence intervals, this is a reduction of between $44.29 \%$ and $77.07 \%$ (mean: $63.44 \%$ ). This is further strengthened by a very large effect size of $\operatorname{eta}_{\mathrm{p}}{ }^{2}=0.784$. We analyzed the influence of the target widths in more detail (see Fig. 4). We can see that the difference is especially apparent for the 20- and 40-pixel targets (pair-wise comparison significant, $\mathrm{p}=0.000$ ), while it is nonsignificant for the 80-pixel targets $(\mathrm{p}=0.653)$. The presentation sequence of the pointing techniques actually had a significant effect on error rate $(F(1,18)=9.396, p=0.001)$. However, detailed analysis showed that it influenced the results in favor of the absolute pointing technique - while the Adaptive Pointing technique significantly decreased in performance when presented second (error rate: $7.22 \%$ compared with $3.73 \%, \mathrm{~F}(1,20)=8.256, \mathrm{p}=0.009)$, the absolute condition benefited (error-rate: $11.08 \%$ compared with $18.79 \%, \mathrm{~F}(1,20)=19.304, \mathrm{p}=0.000)$. Our results therefore tend to show a lower bound of the actual difference.
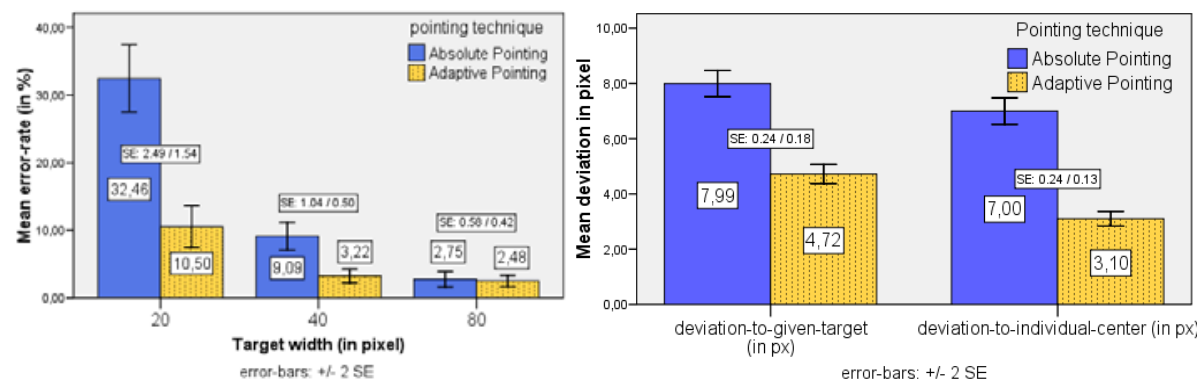

Fig. 4. Comparing error rate (bubble test, left) and dwelling deviations (dwelling test, right)

The dwelling task showed similar results. While users could point to the target with a mean deviation of 4.72 pixels when using the Adaptive Pointing technique, they only managed a mean deviation of 7.99 pixels with absolute pointing (see Fig. 4 on the right, main effect of pointing technique: $F(1,23)=63.191, p=0.000)$. Since we used a 20-pixel target and not just a single dot we assumed participants might not have tried to point to the center but instead just to stay within the boundaries of the target. We therefore calculated the individual center of the pointing a-posteriori for each participant $\times$ trial and the deviation around this center. Results are similar again (Fig. 4 right, 3.1 px vs. 7.0 px, $F(1,23)=119.559$, p=0.000). In short, both the bubble test and the dwelling test strongly support the accuracy hypothesis. 
$\mathrm{H} 2$ : moving: The second hypothesis stated a decreased movement time for the Adaptive Pointing technique only for small targets due to the fact that this measurement also includes clicking on a target. Looking at the results shows that it did indeed take participants only 1.49 seconds to reach a target and click on it when using Adaptive Pointing compared with 1.84 seconds for absolute pointing. Accordingly, an RMANOVA shows a large main effect for pointing technique $(F(1,23)=58.468, p=0.000$, $\operatorname{eta}_{\mathrm{p}}{ }^{2}=0.736$ ). Again, we analyzed the width $\times$ pointing technique interaction in detail to see the influence of the different target widths (see Fig. 5, left). This time, the differences between the two techniques remain significant (pair-wise comparisons, $\mathrm{p}=0.000$ ) in all cases - the size of the effect, however, decreases with increasing target width. This is in line with the results of the previous hypothesis in that the benefit of Adaptive Pointing is particularly evident when having to click on small objects. To sum up, the results clearly support the stated hypothesis and show that Adaptive Pointing is more efficient even for larger target sizes of 80px $(89.6 \mathrm{~mm})$.
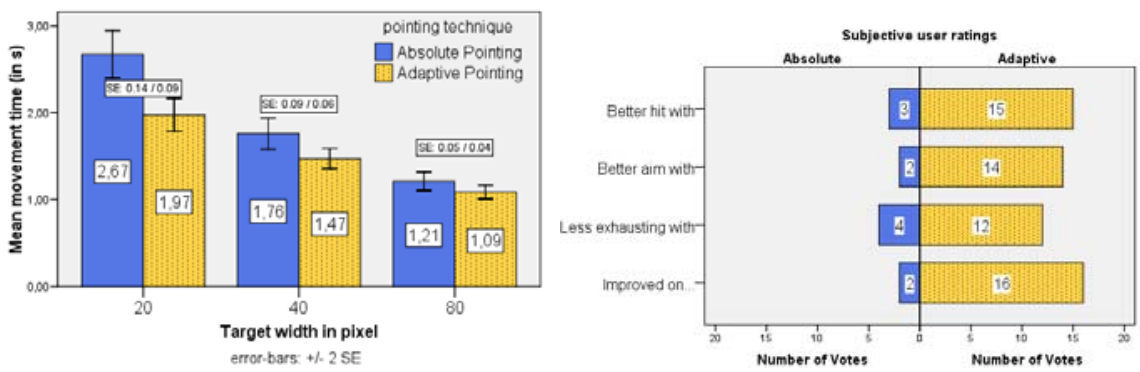

Fig. 5. Comparing movement times (left) and subjective user ratings (right)

H3: imperceptibility: The third hypothesis stated that users would not recognize the change in pointing technique during the experiment or at least would not ascribe it to the laser-pointer itself. Only three users did not recognize any change at all, clearly contradicting the first part of the hypothesis. The remaining 21 participants filled in a questionnaire asking them to define the change experienced during the experiment in more detail by agreeing with statements such as 'Usage got more/less tiresome', 'It was easier/harder to hit the targets', or 'I got better/worse'. Because we had varied the presentation sequence of the pointing techniques, we had a positive and a negative version of each statement. It is important to note that users could choose freely from the list of statements and were not asked to answer each of them. For analysis we then counted how many positive statements a technique received for each question. Negative statements were transformed into positive points for the competing technique. The resulting Fig. 5 (right) reveals that users clearly assigned the positive statements to the Adaptive Pointing technique. A $\mathrm{Chi}^{2}$ test shows that the difference in distribution of the statements between techniques is significant for each case (getting better: $\mathrm{X}^{2}(1, \mathrm{~N}=18)=10.889, \mathrm{p}=0.001$; less tiresome: $\mathrm{X}^{2}(1, \mathrm{~N}=16)=4.0, \mathrm{p}=0.046$; easier to aim: $\mathrm{X}^{2}(1, \mathrm{~N}=16)=9.0, \mathrm{p}=0.003$; easier to hit: $\left.\mathrm{X}^{2}(1, \mathrm{~N}=18)=8.0, \mathrm{p}=0.005\right)$. When asked what might be responsible for the change that they had recognized, participants gave fatigue as the reason for getting worse ( 7 times) while changes to the laser-pointer itself 
(7 times), results of practice (4 times) or an improvement in mental concentration (2 times) were made responsible for getting better. In addition to the above-mentioned statements, the 21 users who noticed a change had to give a total rating on a six-point scale, stating whether the change was for the worse or for the better. We transformed this scale so as ratings higher than 3.5 favored the Adaptive Pointing while values lower than 3.5 rated it as worse. The mean rating was 4.67 with a std. deviation of 1.197. A one-sample t-test (two-sided) reveals that this is a significant difference to the 3.5 test value $(\mathrm{t}(20)=4.466, \mathrm{p}=0.000)$.

To sum up this hypothesis, our initial concern, namely that recognition would mean that the laser-pointer behaved unnaturally, turned out to be wrong. Our participants clearly ascribed positive characteristics to the Adaptive Pointing technique and rated it as significantly better compared with absolute pointing.

\section{Discussion and Conclusion}

The experiment provided some clear-cut results. In every single aspect, the Adaptive Pointing technique proved to be significantly better than a Kalman filter enhanced absolute pointing. We observed a mean reduction in error rate (effectiveness) of about $63 \%$, an improvement in dwelling deviation of between $40 \%$ and $55 \%$, as well as more efficient usage in terms of movement time (19\% mean difference). Furthermore, users stated that they clearly preferred the Adaptive Pointing and assigned positive characteristics such as "better hitting" or "less exhausting" to it. Putting the results in perspective, we can for example compare the dwelling results with the study of Myers et al. [2]. In a similar setting they noted a deviation of between $7.3 \mathrm{~mm}$ and $8.9 \mathrm{~mm}$, which corresponds approximately to our observed deviation of $7.99 \mathrm{px}(=8.95 \mathrm{~mm})$ for absolute pointing, while Adaptive Pointing enabled a deviation of only 4.72px $(=5.29$ $\mathrm{mm})$. With regards to efficiency and effectiveness, former approaches suffered a clear speed-accuracy trade-off [3], while our Adaptive Pointing performed better in both aspects. To conclude, we would like to cite our participants, firstly on the behavior of Adaptive Pointing: "No, it was no big readjustment by any means. It [Adaptive Pointing] was very helpful and happened without any problems. By itself."(ID12), and secondly on its effect: "In the beginning [absolute pointing] I found it exhausting but towards the end [Adaptive Pointing] I almost found it boring. Because then you hit almost every time." (ID24).

\section{References}

1. Hinckley, K.: Input technologies and techniques. In: Handbook of Human-Computer Interaction, pp. 161-176 (2008)

2. Myers, B.A., Bhatnagar, R., Nichols, J., et al.: Interacting at a distance: measuring the performance of laser-pointers and other devices. In: Proc. CHI 2002, pp. 33-40 (2002)

3. Vogel, D., Balakrishnan, R.: Distant freehand pointing and clicking on very large, high resolution displays. In: Proc. UIST 2005, pp. 33-42 (2005)

4. Oh, J., Stuerzlinger, W.: Laser-pointers as collaborative pointing devices. In: Proc: GI $2002(2002)$ 
5. Vaillancourt, E., Newell, K.M.: Amplitude changes in the 8-12, 20-25, and 40 hz oscillations in finger tremor. Clinical neurophysiology 111(10), 1792-1801 (2000)

6. Balakrishnan, R., MacKenzie, I.S.: Performance differences in the fingers, wrist, and forearm in computer input control. In: Proc. CHI 1997, pp. 303-310 (1997)

7. Card, S.K., Mackinlay, J.D., Robertson, G.G.: A morphological analysis of the design space of input devices. ACM Trans. Inf. Syst., 99-122 (1991)

8. Gibbs, B.: Controller design: Interactions of controlling limbs, time-lags and gains in positional and velocity systems. Ergonomics, 385-402 (1962)

9. Worden, A., Walker, N., Bharat, K., Hudson, S.: Making computers easier for older adults to use: area cursors and sticky icons. In: Proc. CHI 1997, pp. 266-271 (1997)

10. Cockburn, A., Firth, A.: Improving the acquisition of small targets. In: Proc. British Computer Society Conference on HCI 2003, pp. 181-196 (2003)

11. Baudisch, P., Cutrell, E., Hinckley, K., Eversole, A.: Snap-and-go: helping users align objects without the modality of traditional snapping. In: Proc. CHI 2005, pp. 301-310 (2005)

12. Blanch, R., Guiard, Y., Beaudouin-Lafon, M.: Semantic pointing: improving target acquisition with control-display ratio adaptation. In: Proc. CHI 2004, pp. 519-526 (2004)

13. Forlines, C., Vogel, D., Balakrishnan, R.: Hybridpointing: fluid switching between absolute and relative pointing with a direct input device. In: Proc. UIST 2006, pp. 211-220 (2006)

14. Meyer, D.E., Abrams, R.A., Kornblum, S., Wright, C.E., Smith, J.E.K.: Optimality in human motor performance: Ideal control of rapid aimed movements. Psychological Review, 340-370 (1988)

15. Balakrishnan, R.: "beating” fitts' law: virtual enhancements for pointing facilitation. Int. J. Hum.-Comput. Stud., 857-874 (2004)

16. Microsoft. Pointer ballistics for windows xp (2008), http: / /www. microsoft.com/whdc/archive/pointer-bal.mspx

17. Casiez, G., Vogel, D., Balakrishnan, R., Cockburn, A.: The impact of control-display gain on user performance in pointing tasks. Human-Computer Interaction 23(3) (2008)

18. Frees, S., Kessler, G.D., Kay, E.: PRISM interaction for enhancing control in immersive virtual environments. ACM Trans. Comput.-Hum. Interact. 14(1) (2007)

19. McGuffin, M., Balakrishnan, R.: Acquisition of expanding targets. In: Proc. CHI 2002, pp. 57-64 (2002)

20. Baudisch, P., Cutrell, E., Robbins, D., Czerwinski, M., Bederson, P.T.B., Zierlinger, A.: Drag-and-pop and drag-and-pick: Techniques for accessing remote screen content on touch- and pen-operated systems. In: Proc. Interact 2003, pp. 57-64 (2003)

21. König, W.A., Bieg, H.-J., Schmidt, T., Reiterer, H.: Position-independent interaction for large high-resolution displays. In: Proc. IHCI 2007 (2007)

22. Bieg, H.-J.: Laserpointer and eye gaze interaction - design and evaluation. Master's thesis, University of Konstanz (2008),

http://hci.uni-konstanz.de/intehrdis/Bieg2008.pdf

23. Sutter, C., Muesseler, J., Bardos, L., Ballagas, R., Borchers, J.: The impact of gain change on perceiving one's own actions. In: Proc. M\&C 2008 (2008) 